\title{
$\mathrm{Rh}(\mathrm{III})$ 催化不对称[3+2]环化构建兼有轴手性和中心手性的狮类化合物
}

\author{
袁文揆史炳锋* \\ (浙江大学化学系 杭州 310027)
}

\section{Rh(III)-Catalyzed Asymmetric [3+2] Annulative Construction of Axially and Centrally Chiral Indenes}

\author{
Yuan, Wenkui \\ Shi, Bingfeng* \\ (Department of Chemistry, Zhejiang University, Hangzhou 310027)
}

近年来, 过渡金属催化的不对称碳氢键活化策略在 手性化合物的高效合成中取得了巨大的进展 ${ }^{[1-7]}$. 然而, 不对称碳氢键官能化反应主要集中于单一手性中心的 产生，同时构建具有多个手性中心的策略更加具有吸引 力, 也更具挑战性. 迄今为止, 利用不对称碳氢键活化 策略同时构建双手性化合物常见于芳烃碳氢键活化后 对烯烃的立体选择性插入, 从而产生具有两个相邻手性 中心的分子(Scheme 1a). 或者是碳氢键官能化产生第一 个手性中心进而诱导第二个手性中心产生(Scheme 1b). 亦或者通过芳烃的去对称化的形成第一个手性中心, 随 后碳氢键官能化给出另一个手性中心(Scheme 1c). 这些 反应的共同特点是两个手性中心以相互关联方式创建 的. 因此, 当两个手性中心之间远离且没有相互作用时, 在单一反应中使用相同的手性催化剂产生两个分立的 手性中心是极具挑战性的.

为了解决这一难题, 陕西师范大学化学化工学院李 兴伟课题组 ${ }^{[8]}$ 提出了一种新的催化策略, 其基本原理是 在催化循环中容纳两个不同的立体决定步骤, 具体是应 用带有亲电导向基团的芳烃底物和炔反应实现碳氢键 活化进而[3+2]环化产生轴手性和中心手性的苑类化合 物(Scheme 1d). 目前, 手性 Rh(III)催化的不对称碳氢键 活化反应已有诸多报道 ${ }^{[9-10]}$, 然而带有亲电导向基的芳 烃的偶联却很少被报道, 并且仅限于中心手性的构建. 因此该策略需要解决几个挑战: 首先, 催化剂应传达适 当的手性信息, 以适应两个不相关的立体迁移插入过 程. 特别是, 需要足够的导向基-炔烃相互作用来确保 初始(轴向)手性识别. 其次, 产物中狮基含有带有酸性 的差向异构手性中心, 温和的反应条件显得很重要. (a) Difunctionalization of $\mathrm{C}=\mathrm{C}$ bonds: generation of correlated and adjacent chiral centers

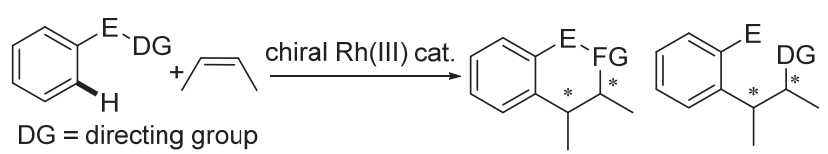

$D G=$ directing group

(b) Sequention formation og two chiral centers: chiral Induction toward the 2 nd chiral center

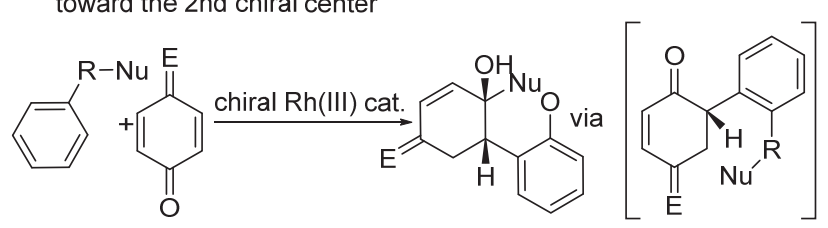

(c) Desymmertrization for creation of central \& axial chirality

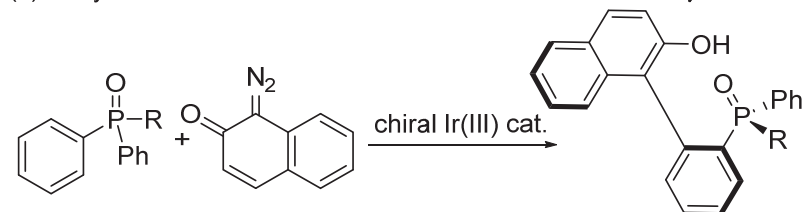

(d) Construction of distally dosposed axial and central chirality via two uncorrelated stereo-determining steps

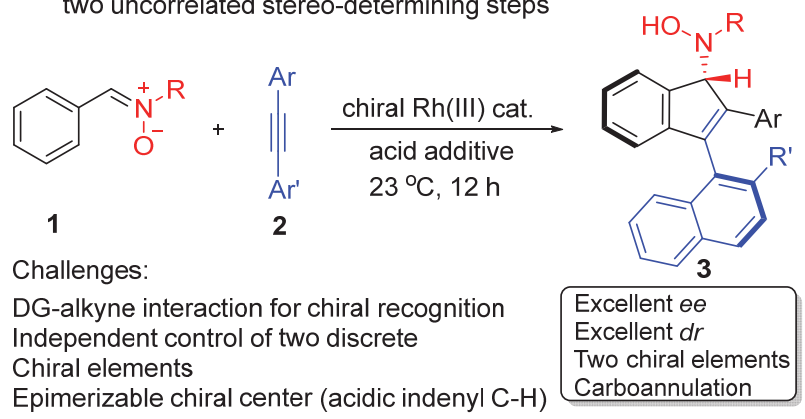

图式 1 碳氢键官能化合成多手性中心策略

Scheme 1 Formation of multiple chiral elements via $\mathrm{C}-\mathrm{H}$ functionalization

由于两个不同的立体决定步骤被认为是互不影响

* Corresponding author. E-mail: bfshi@zju.edu.cn. Published online August 21, 2021. 
的，作者首先使用 Rh-5 实现只具有中心手性狮 3 的合 成(Scheme 2a, 90\% 99\% ee). 之后作者对反应体系微 调, 使用 Rh-1 实现了兼具联(杂)芳轴手性和中心手性产 物 5 (Scheme 2b, 88\% 98\% ee) 以及碳氮轴手性和中心 手性产物 7 (Scheme 2c, 91\% 95\% ee) 的合成. 反应体 系具有优秀的底物普适性和立体选择性.
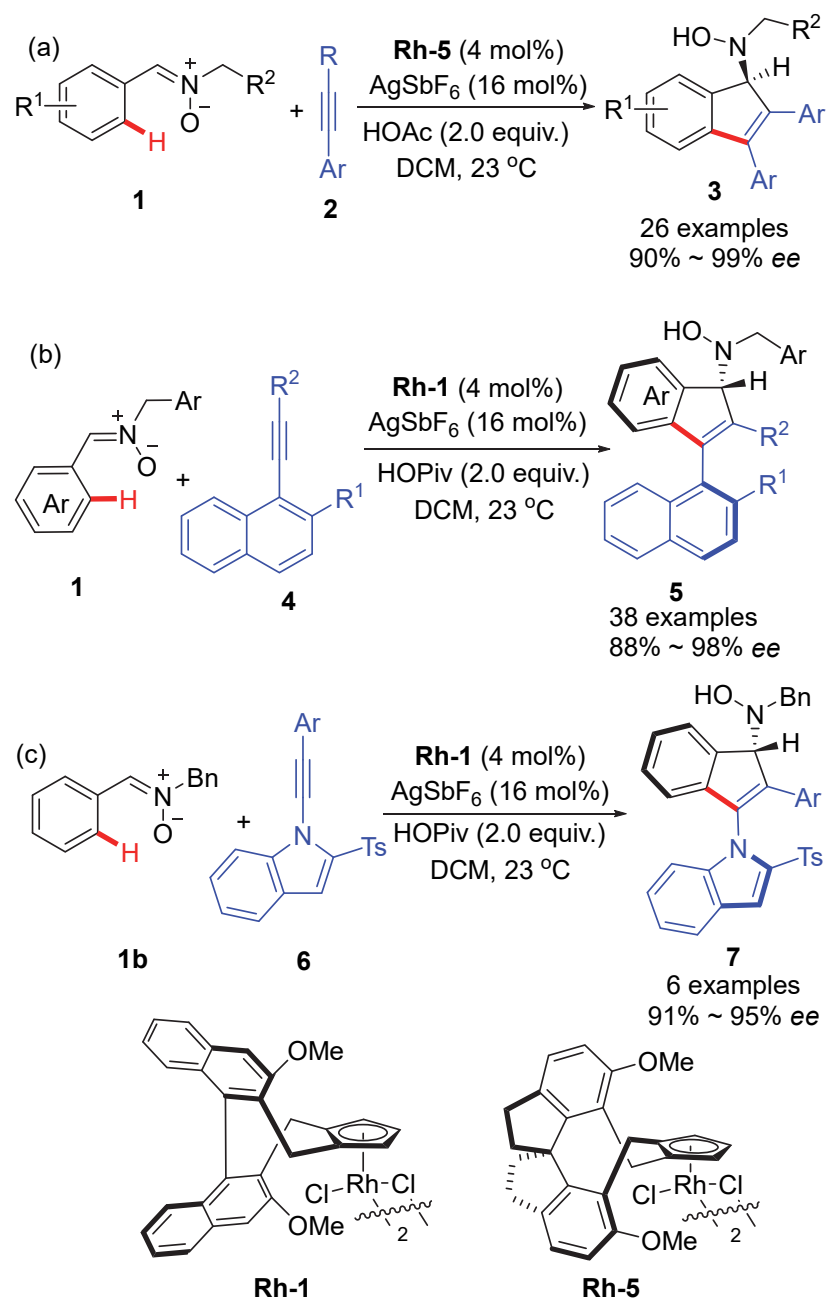

图式 2 手性荫的底物范围

Scheme 2 Scope of chiral indenes

值得注意的是, 当 $N$-叔丁基硝酮被用于反应时, 只 能以 $88 \% e e$ 获得 $\mathrm{C}-\mathrm{N}$ 轴手性䒢酮 8 (Scheme 3).

随后, 作者对偶联产物进行衍生化研究(Scheme 4). $5 \mathbf{a}$ 和 $5 \mathbf{b}$ 在 $\mathrm{Cu}(\mathrm{OAc})_{2}$ 催化氧化下, $\mathrm{C}-\mathrm{N}$ 裂解以中等产 率得到轴手性狮酮 $9 a$ 和 $9 b$. 5 c 脱保护得到 2-䒺酚 10. 3a 经 IBX 氧化以 $65 \%$ 得到硝酮 $11.3 \mathrm{a}$ 经乙酰化保护, 随后 $\mathrm{N}-\mathrm{O}$ 键的还原裂解，以高产率生成脱氧茚胺 $\mathbf{1 2}$.

作者也研究了反应的动力学同位素效应, 以单手性 产物 3a/3a- $d_{4}$ 测得 $k_{\mathrm{H}} / k_{\mathrm{D}}=3.8$, 双手性产物 $5 \mathbf{a} / \mathbf{5} \mathbf{a}-d_{4}$ 测得 $k_{\mathrm{H}} / k_{\mathrm{D}}=3.3$. 说明碳氢键断裂可能是反应的决速步骤.

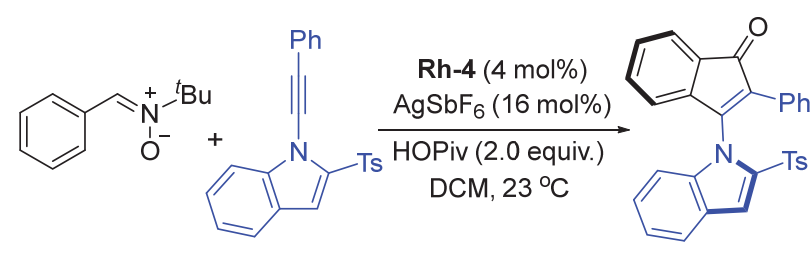

$6 b$

8, $88 \%$ ee

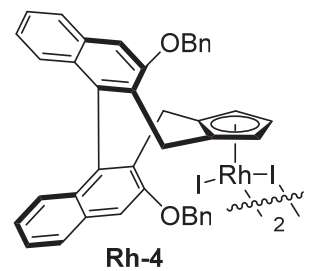

图式 $3 \mathrm{C}-\mathrm{N}$ 轴手性狮酮的合成

Scheme 3 Synthesis of a $\mathrm{C}-\mathrm{N}$ axially chiral indenone

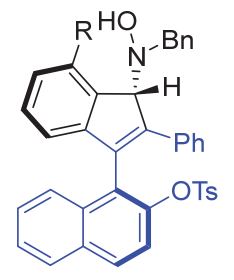

5a, $\mathrm{R}=\mathrm{H}, 96 \%$ ee 5b, R = F, 95\% ee<smiles>CC(=O)Oc1ccc2ccccc2c1C1=C(c2ccccc2)[C@@H](N(O)Cc2ccccc2)c2ccccc21</smiles>

5c, $98 \%$ ee
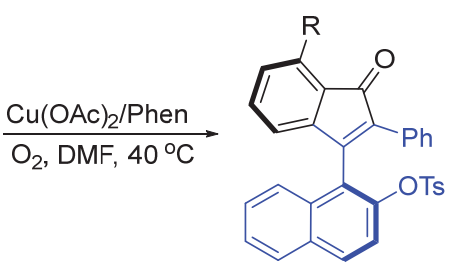

9a, $\mathrm{R}=\mathrm{H}, 59 \%$ yield, $91 \%$ ee $9 \mathbf{b}, \mathrm{R}=\mathrm{H}, 64 \%$ yield, $93 \%$ ee

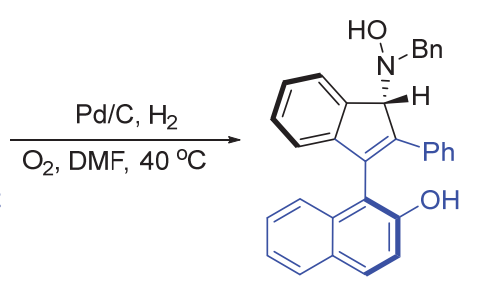

$10,60 \%$ yield, $98 \%$ ee

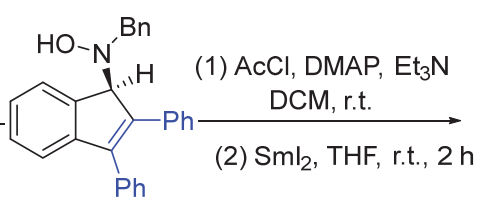

3a, $99 \%$ ee<smiles>c1ccc(CN[C@H]2C(c3ccccc3)=C(c3ccccc3)c3ccccc32)cc1</smiles>

$12,88 \%$ yield, $97 \%$ ee

图式 4 偶联产物的衍生化反应

Scheme 4 Derivatization of coupled products

作者经密度泛函理论(DFT)计算研究认为中间体 $\mathbf{A}$ 热力学最稳定, 随后的炔键插入更加符合动力学要求, 从而生成 $S$-型烯基中间体 $\mathbf{C}$. 中间体 $\mathbf{C}$ 的烯基经 $(R e)-$ 面选择性迁移插入到硝酮中, 该步骤决定了反应的非对 
映选择性. 最后, $\mathrm{Rh}-\mathrm{O}$ 键经质子解生成具有轴和中心 手性的手性产物(Scheme 5a). 在中间体 $\mathbf{A}$ 中炔基苯环与 OTs 苯环存在 $\pi-\pi$ 堆积作用，降低 OTs 和硝酮基之间的 空间排斥作用. 作者也通过控制实验验证了机理的合理 性，当邻苯基硝酤(1e)用于反应时产物 $5 \mathrm{e}$ 仅以 $65 \% \mathrm{ee}$ 获得. 说明与邻位 $\mathrm{Br}$ 和 $\mathrm{CF}_{3}$ 的硝酮底物 $(\mathbf{1 c}, 1 \mathrm{~d})$ 相比, 邻 位苯基与菜基的 $\pi-\pi$ 堆积作用使中间体 $\mathbf{A}^{\prime}$ 更加稳定，从
而使反应的不对称选择性降低(Scheme 5b).

综上所述，李兴伟课题组报道了手性 Rh(III)催化硝 酮与炔烃的不对称 $[3+2]$ 环化反应, 在手性 $\mathrm{Rh}(\mathrm{III})$ 催化 剂控制下，通过两个不同的立体决定步骤连续产生轴手 性和中心手性，该反应策略可用于高附加值芳烃的合 成, 也为不对称碳氢键活化的发展提供了新的思路.

(a) Two distinct enantio-determining steps toward formation of major chiral product

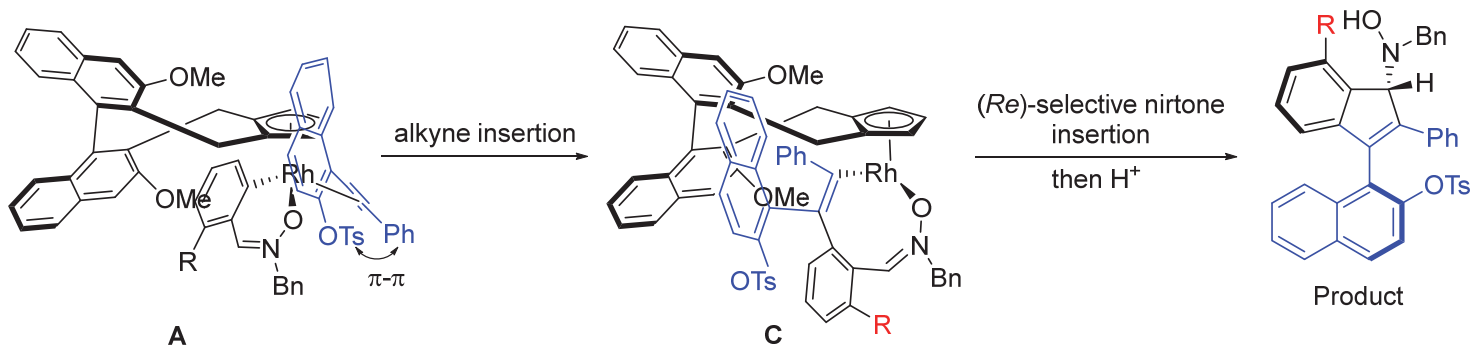

(b) Mechanistic control experiments

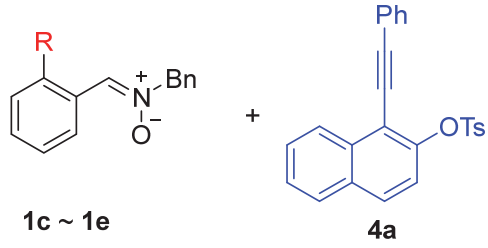

$1 \mathrm{c} \sim 1 \mathrm{e}$

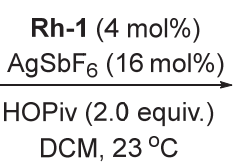

DCM, $23^{\circ} \mathrm{C}$

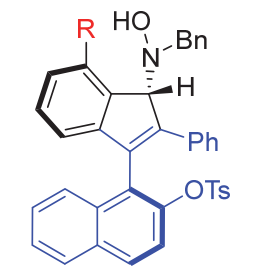

5c, $\mathrm{R}=\mathrm{Br}, 95 \%$ ee

$5 d, \mathrm{R}=\mathrm{CF}_{3}, 88 \%$ ee

$5 e, \mathrm{R}=\mathrm{Ph}, 65 \%$ ee

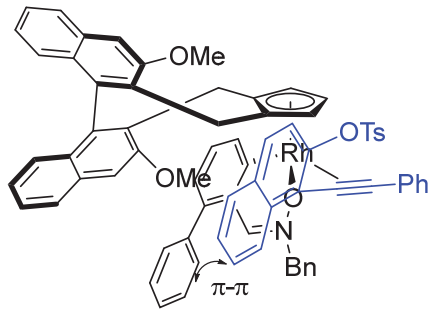

$A^{\prime}$

图式 5 反应立体决定步骤及机理

Scheme 5 Stereo-determining steps and mechanistic considerations

\section{References}

[1] Newton, C. G.; Wang, S.-G.; Oliveira, C. C.; Cramer, N. Chem. Rev. 2017, 117, 8908 .

[2] Saint-Denis, T. G.; Zhu, R.-Y.; Chen, G.; Wu, Q.-F.; Yu, J.-Q. Science 2018, 359, No. eaao4798

[3] Zhang, H.; Gu, Q.; You, S.-L. Chin. J. Org. Chem. 2019, 39, 15 (in Chinese). (张慧君, 顾庆, 游书力, 有机化学, 2019, 39, 15.)

[4] Liao, G.; Zhang, T.; Lin, Z.-K.; Shi, B.-F. Angew. Chem., Int. Ed. 2020, 59, 19773.

[5] Shao, Q.; Wu, K.; Zhuang, Z.; Qian, S.; Yu, J.-Q. Acc. Chem. Res.
2020, 53, 833 .

[6] Zhang, S.; Liao, G.; Shi, B.-F. Chin. J. Org. Chem. 2019, 39, 1522 (in Chinese). (张硕，廖港，史炳锋，有机化学， 2019, 39, 1522.)

[7] Wang, Q.; Gu, Q.; You, S.-L. Acta Chim. Sinica 2019, 77, 690 (in Chinese). (王强, 顾庆, 游书力, 化学学报, 2019, 77, 690.)

[8] Wang, F.; Jing, J.; Zhao, Y.; Zhu, X.; Zhang, X.-P.; Zhao, L.; Hu, P.; Deng, W.-Q.; Li, X. Angew. Chem., Int. Ed. 2021, 60, 16628.

[9] Ye, B.; Cramer, N. Acc. Chem. Res. 2015, 48, 1308.

[10] Wang, Q.; Liu, C.-X.; Gu, Q.; You, S.-L. Sci. Bull. 2021, 66, 210.

(Lu, Y.) 